\title{
Bacteriological Profile and Antibiogram of Aerobic Blood Culture Isolates from Intensive Care Units in a Teaching Tertiary Care Hospital
}

\author{
${ }^{1}$ Ashima Katyal, ${ }^{2}$ Deepinder Singh, ${ }^{3}$ Madhu Sharma, ${ }^{4}$ Uma Chaudhary
}

\begin{abstract}
Context: Infection rates in the intensive care unit (ICU) have been found to be the highest among all hospital-acquired infections.
\end{abstract}

Aim: To determine the prevalence of current bacteriological profile in blood cultures along with their antibiogram from ICU patients.

Settings and design: The present study was conducted in the Department of Microbiology, over a period of 1 year, i.e., from September 2016 to August 2017.

Materials and methods: A total of 2,028 blood cultures were received from various ICUs. The isolates were identified by standard microbiological procedures, and antibiogram was determined by Clinical and Laboratory Standards Institute (CLSI) guidelines.

Statistical analysis: Qualitative data were presented as mean and standard deviation and quantitative data as proportions. Association was tested using chi-squared test. Statistical significance was considered when $p<0.05$. Statistical Package for the Social Sciences version 20.0 software was used for analysis.

Results: Total positive cultures were obtained in 504 (24.86\%) cases. Among the Gram-positive (GP) isolates 288 (57.14\%), coagulase-negative Staphylococci (CoNS) $55.5 \%$ was the most common followed by Staphylococcus aureus $34 \%$ and Enterococcus spp. $10.4 \%$. Acinetobacter baumannii $52.3 \%$ was the most common Gram-negative (GN) isolate, 216 (42.85\%), followed by Escherichia coli $27.7 \%$, Klebsiella pneumoniae $14.35 \%$, and Pseudomonas aeruginosa 5.5\%. Most of the GP isolates were susceptible to vancomycin and linezolid and GN isolates to imipenem and meropenem.

Conclusion: This study shows the high number of positive blood cultures in ICUs, which can be attributed to increasing use of intravenous devices, interventions, and immunosuppression. Hence, this study may be a useful guide for initiating early empiric therapy for ICU patients and will help in formulation of antibiotic policy in our institute.

\footnotetext{
${ }^{1}$ Senior Resident, ${ }^{2}$ Postgraduate Student, ${ }^{3}$ Professor, ${ }^{4}$ Senior Professor and Head

${ }^{1-4}$ Department of Microbiology, Pt. Bhagwat Dayal Sharma Post Graduate Institute of Medical Sciences, Rohtak, Haryana, India

Corresponding Author: Ashima Katyal, Senior Resident Department of Microbiology, Pt. Bhagwat Dayal Sharma Post Graduate Institute of Medical Sciences, Rohtak, Haryana, India Phone: +919466676878, e-mail: docak07@gmail.com
}

Keywords: Bloodstream infections, Intensive care unit, Multidrug resistant.

How to cite this article: Katyal A, Singh D, Sharma M, Chaudhary U. Bacteriological Profile and Antibiogram of Aerobic Blood Culture Isolates from Intensive Care Units in a Teaching Tertiary Care Hospital. J Health Sci Res 2018;9(1):6-10.

Source of support: Nil

Conflict of interest: None

\section{INTRODUCTION}

Bloodstream infection (BSI) is a devastating, preventable infectious complication in critical care units. The impacts of BSIs have far-reaching consequences, resulting in increased mortality rate, prolonged stay in ICUs, and high cost to the individual. ${ }^{1}$ The crude mortality rate in Indian surveys ranged from 35.2 to $44.9 \%$, which is quite higher as compared with the developed nations like the Untied States. ${ }^{2}$ It is alarming that $30 \%$ patients of BSI receive inappropriate empirical therapy, which further adds to their poor outcome. . $^{3,4}$

Studies have shown that there is a wide range of bacteria, both GP and GN, which are associated with these infections. The advent of antimicrobial resistance among ICU microorganisms is another issue of public health concern. ${ }^{5-8}$ The diagnosis for such infections can be made with blood cultures, which has a high positive predictive value. Early diagnosis and start of appropriate antibiotic therapy can reduce the burden of BSI in ICU.

Therefore, this study was designed to know the current spectrum of aerobic blood culture isolates from ICU patients and the trend of resistance prevalent among these isolates in our institute.

\section{MATERIALS AND METHODS}

\section{Study Design}

Ours was a hospital-based retrospective study carried out in a teaching tertiary care hospital, India. The data were collected by reviewing the records of 2,028 patients admitted to various ICUs of the hospital from September 2016 to August 2017. 


\section{Setting}

Various ICUs of a teaching tertiary care center in India.

\section{Patient Data}

The blood samples from these patients were routinely processed for blood culture in the Department of Microbiology. Data collection included age and sex of the patients, the results of the blood culture, and antimicrobial susceptibility testing (AST).

\section{Blood Culture}

Blood samples from each patient were collected taking all aseptic precautions. For all the samples, phlebotomy was performed after achieving antisepsis of vein puncture site with $70 \%$ alcohol followed by $2 \%$ tincture iodine. About 5 to $10 \mathrm{~mL}$ of blood from adults and $2 \mathrm{~mL}$ for pediatric age group was collected, which was then inoculated in brain heart infusion broth of 50 and $10 \mathrm{~mL}$ respectively. Blood culture bottles were incubated at $37^{\circ} \mathrm{C}$ aerobically for 24 hours followed by subcultures on blood agar and MacConkey agar. Blood culture bottles that do not show signs of growth (turbidity or hemolysis) were again subcultured on $2 \mathrm{nd}$, 3rd, and 7 th day and were reported negative on 7 th day after final subculture. Isolates were identified by standard microbiological procedures including Gram's stain, colony morphology, and biochemical reactions. ${ }^{9}$ These were also confirmed by automated identification and antimicrobial susceptibility system, i.e., B.D. Phoenix. The results of AST were interpreted as per CLSI guidelines 2016. ${ }^{10}$ The multidrug resistance (MDR) was defined as resistance to at least one agent in three or more categories of antimicrobial agents. ${ }^{11}$

\section{RESULTS}

A total of 2,028 blood samples of the patients suspected of bacteremia, admitted to critical care units of the hospital, were routinely processed for blood culture in the Department of Microbiology from September 2016 to August 2017. Out of these patients, 1,258 (62.03\%) were males. Male:female ratio was approximately 1.6:1. Median age of the patient was 34 years with range of 1 day to 85 years. The predominant sources of blood culture samples were neonatal ICU (NICU) 1,716 (84.6\%),
Table 1: Distribution of microorganisms according to source of isolation

\begin{tabular}{lllll}
\hline Organisms & $\begin{array}{l}N I C U \\
(n=432)\end{array}$ & $\begin{array}{l}R I C U \\
(n=60)\end{array}$ & $\begin{array}{l}P I C U \\
(n=12)\end{array}$ & $\begin{array}{l}\text { Total } \\
(n=504)(\%)\end{array}$ \\
\hline CoNS & 144 & 14 & 2 & $160(31.7)$ \\
S. aureus & 96 & 0 & 2 & $98(19.4)$ \\
Enterococcus spp. & 24 & 6 & 0 & $30(5.9)$ \\
E. coli & 60 & 0 & 0 & $60(11.9)$ \\
K. pneumoniae & 12 & 11 & 8 & $31(6.15)$ \\
A. baumannii & 84 & 29 & 0 & $113(22.4)$ \\
P. aeruginosa & 12 & 0 & 0 & $12(2.3)$ \\
\hline
\end{tabular}

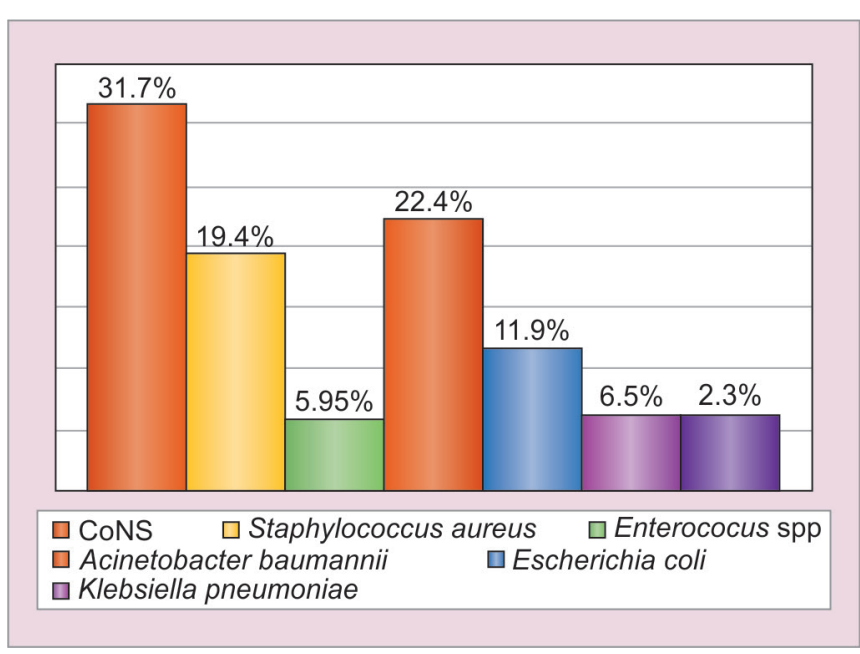

Graph 1: Distribution of microorganisms isolated from blood cultures

followed by respiratory ICU 276 (13.6\%), and pediatrics ICU 36 (1.77\%). Total positive cultures in the present study were obtained in 504 (24.86\%) cases. Among the culturepositive isolates, 288 (57.14\%) were GP and 216 (42.85\%) were GN. The most common isolate was CoNS 160 (31.7\%) followed by A. baumannii 113 (22.4\%) and S. aureus 98 (19.4\%) (Table 1 and Graph 1). All the BSIs in the present study were due to single organism only.

The antibiotic susceptibility profile of blood culture isolates is depicted in Table 2 (for GP isolates) and Table 3 (for GN isolates). Among the GP bacterial isolates, 100\% of CoNS, $84 \%$ of Enterococcus spp., and $82 \%$ of S. aureus were resistant to penicillin group of antibiotics. However, most of the GP isolates were susceptible to vancomycin, linezolid, and clindamycin.

Among the GN isolates, Acinetobacter spp. (52.3\%) and E. coli $(27.7 \%)$ were dominant species. Third-generation

Table 2: Antimicrobial susceptibility profile of GP isolates

\begin{tabular}{llllllll}
\hline Isolates & $E$ & $P$ & $C X$ & Co & $C D$ & LZ & VA \\
\hline CoNS $(\mathrm{n}=160)$ & $17(10.62 \%)$ & 0 & $78(48.75 \%)$ & $54(33.75 \%)$ & $115(71.87 \%)$ & $156(97.5 \%)$ & NA \\
S. aureus $(\mathrm{n}=98)$ & $22(22.44 \%)$ & $18(18.36 \%)$ & $67(68.36 \%)$ & $67(68.36 \%)$ & $88(89.79 \%)$ & $95(96.93 \%)$ & NA \\
Enterococcus spp. $(\mathrm{n}=30)$ & $10(33.33 \%)$ & $5(16.66 \%)$ & $12(40 \%)$ & $19(63.33 \%)$ & $23(76.66 \%)$ & $29(96.66 \%)$ & $28(93.33 \%)$ \\
\hline
\end{tabular}

E: Erythromycin; P: Penicillin; Cx: Cefoxitin; Co: Cotrimoxazole; CD: Clindamycin; Lz: Linezolid; Va: Vancomycin; NA: Not applicable; According to CLSI guidelines 2017 routine testing with vancomycin disk is no more recommended for Staphylococcus spp. 
Table 3: Antimicrobial susceptibility profile of GN isolates

\begin{tabular}{|c|c|c|c|c|c|c|c|c|c|}
\hline \multirow[b]{2}{*}{ Isolate $(n=216)$} & \multicolumn{9}{|c|}{ Antibiotic susceptibility pattern (\%) } \\
\hline & $A K$ & $A C$ & $G$ & PIT & CIP & $D O$ & COT & IMP & $M R P$ \\
\hline E. coli $(\mathrm{n}=60)$ & 0 & $11(18.3 \%)$ & $24(40 \%)$ & $29(48.3 \%)$ & $38(63.3 \%)$ & $18(30 \%)$ & $45(75 \%)$ & $55(91.6 \%)$ & $54(90 \%)$ \\
\hline K. oxytoca $(\mathrm{n}=31)$ & $15(48.4 \%)$ & $8(25.8 \%)$ & $12(38.7 \%)$ & $11(35.5 \%)$ & $19(61.3 \%)$ & $12(38.7 \%)$ & $23(74.2 \%)$ & $27(87.1 \%)$ & $25(80.6 \%)$ \\
\hline $\begin{array}{l}P . \text { aeruginosa } \\
(n=12)\end{array}$ & $4(33.3 \%)$ & $1(8.3 \%)$ & $5(41.6 \%)$ & $4(33.3 \%)$ & $6(50 \%)$ & $9(75 \%)$ & $9(75 \%)$ & $8(66.6 \%)$ & $11(91.6 \%)$ \\
\hline $\begin{array}{l}\text { Acinetobacter spp. } \\
(\mathrm{n}=113)\end{array}$ & $28(24.7 \%)$ & $12(10.6 \%)$ & $10(8.8 \%)$ & $16(14.2 \%)$ & $32(28.3 \%)$ & $57(50.4 \%)$ & $78(69 \%)$ & $99(87.6 \%)$ & $87(76.9 \%)$ \\
\hline
\end{tabular}

Ak: Amikacin; AC: Amoxyclav; G: Gentamicin; PIT: Piperacillin-Tazobactam; CIP: Ciprofloxacin; Do: Doxycycline; COT: Cotrimoxazole; IMP: Imipenem; MRP: Meropenem

cephalosporins showed a very weak activity against them. Carbapenems showed good activity against all the isolates with susceptibility of $87 \%$ for Acinetobacter spp. and Klebsiella oxytoca and $91 \%$ for E. coli and P. aeruginosa. Moreover, $100 \%$ isolates showed susceptibility to colistin and polymyxin B. However, the majority of the isolates were MDR.

\section{DISCUSSION}

Patients admitted to ICUs tend to develop nosocomial BSIs, which lead to increase in morbidity and mortality among these patients. The present study provides information regarding distribution of aerobic bacterial isolates along with their antibiogram in patients admitted to ICUs, which play a crucial role in effective management of these patients. The blood culture positivity rate in our study was $24.86 \%$, which is comparable with various studies from India and abroad. Similar findings were observed in a study done in Ethiopia by Ali and Kebede ${ }^{12}(24.2 \%)$. Studies from India by Gill and Sharma ${ }^{13}(24.8 \%)$, Arora and $\operatorname{Devi}^{14}(20.02 \%)$, and Sharma et $\mathrm{al}^{15}(33.9 \%)$ also showed comparable results. Slight variation may be due to various factors like geographical location, timing, and number of blood cultures or difference in blood culture identification systems.

A wide range of organisms are responsible for BSIs and the same has been reported by various researchers. In the present study, among the culture-positive isolates, 288 (57.14\%) were GP and 216 (42.85\%) were GN. There are various studies from different parts of the world that have previously reported the higher prevalence of Gram-positive bacteria (GPB) over Gram negative bacteria (GNB); a study by Gill and Sharma ${ }^{13}$ showed GP (53\%) preponderance and Wasihun et $\mathrm{al}^{16}$ reported $72.2 \%$ of bacteremia by GPB and $27.8 \%$ by GNB, Dagnew et al ${ }^{17}$ also reported $69 \%$ GPB and 31\% GNB. Among the GP isolates, CoNS 160 (55.5\%) was the most common followed by S. aureus 98 (34\%) and Enterococcus spp. 30 (10.4\%), and this has also been reported from various parts of the country. ${ }^{18-20}$ The higher prevalence of CoNS in the present study could be explained by the fact that most isolates were from the NICU. Although CoNS is a normal skin commensal, but now its pathogenic nature has been well established, especially, in premature infants and with central venous lines. The findings are of significant concern, as in the hospital settings, these are associated with a high degree of antimicrobial resistance and poor patient outcome. $^{13}$

However, on the contrary, many studies from India and other developing countries have reported GNB preponderance in hospitalized patients: studies in India by Gupta and Kashyap ${ }^{21}$ with $58.3 \%$ GNB and $41.65 \%$ GPB, Singh et $\mathrm{al}^{22}$ with $51.82 \%$ GNB and $46.56 \%$ GPB, and a Nigerian study by Nwadioha et $\mathrm{al}^{23}$ with $69.3 \%$ GNB and $30.7 \%$ GPB. The etiologic profile and antibiotic susceptibility pattern vary in different geographical areas due to variations in the social and environmental conditions. The A. baumannii 113 (52.3\%) was the most common GN isolate followed by E. coli $60(27.7 \%)$, K. pneumoniae 31 (14.35\%), and P. aeruginosa 12 (5.5\%).

The irrational use and over-the-counter availability of antibiotics have led to higher prevalence of MDR microorganisms and invasive diseases like sepsis. The antimicrobial resistance profile showed high degree of resistance to GP and GN microorganisms. Most of the isolates $(46.5 \%)$ in our study were MDR, which is in concordance with previous studies. ${ }^{14,17,21}$ Among the GP isolates, methicillin-resistant Staphylococcus aureus was found in significant frequency $(68.36 \%)$, which is quite high as compared with previous studies. Earlier studies have reported increased resistance to erythromycin and penicillin group of antibiotics among GP organisms, a finding also observed in the present study. ${ }^{14,21}$ All the GP organisms showed good susceptibility to clindamycin, linezolid, and vancomycin ranging from 71 to $98 \%$.

Among the GN organisms, overall antibiotic profile suggests high degree of MDR (63.4\%) organisms in our hospital. All the GNB showed poor susceptibility to beta-lactam antibiotics. The fact that the beta-lactam antibiotics are the most commonly prescribed drugs for both inpatients and outpatients could be the reason for such high level of resistance. Both the members of the 
family Enterobacteriaceae showed good susceptibility to ciprofloxacin $(63 \%)$, cotrimoxazole $(75 \%)$, and carbapenems (87\%). Although previous studies have shown good susceptibility to amikacin and combination drugs like cefoperazone/sulbactam, this was not observed in our study. ${ }^{6,21}$ Similarly, nonfermenters in the present study were highly susceptible to carbapenems (85\%), cotrimoxazole (75\%), and doxycycline (70\%). In contrast to previous studies, ${ }^{6,14,18}$ our study observed poor susceptibility of nonfermenters to amikacin (24\%) and piperacillin/tazobactam (20\%) combination. The difference in the antibiotic policy prevailing in various hospitals could be the reason for such discordance.

We observed $24(11.11 \%)$ of the GN isolates were resistant to carbapenems, with 15 (62.5\%) and 9 (37.5\%) isolates among nonfermenters and members of Enterobacteriaceae respectively. The biggest threat with the infections caused by these organisms is the limited antibiotics for available treatment. Worldwide, their incidence is rising with variations due to geological and geographical differences. With the limited options available for treatment and increasing resistance, the clinicians are left with the so-called last resort drugs, i.e., colistin and polymyxin, which could soon lead to the most dreaded condition, viz., pan-drug resistance.

\section{CONCLUSION}

The present study showed the prevalence of aerobic bacteria isolated from critically ill patients in ICUs. The study identified both GP and GN organisms to be responsible for causing bacteremia and most of them were MDR. This implies that blood cultures must always be done in suspected cases of bacteremia and septicemia, and once the susceptibility profile of the organism is known, the de-escalation of the high-end antibiotics should be encouraged to reduce antimicrobial pressure. Moreover, stringent hospital infection policy and good antibiotic policy in the hospital with routine surveillance for baseline resistance will go a long way in combating MDR in pathogens.

\section{REFERENCES}

1. Yu VL, Hansen DS, Ko WC, Sagnimeni A, Kugman KP, Gottberg AV, Goossens H, Wagener MM, Benedi VJ; the International Klebsiella Study Group. Virulence characteristics of Klebsiella pneumoniae and clinical manifestation of $K$. pneumoniae blood stream infections. Emerg Infect Dis 2007 Jul;13(7):986-993.

2. Friedman ND, Kaye KS, Stout JE, McGarry SA, Trivette SL, Briggs JP, Lamm W, Clark C, MacFarquhar J, Walton AL, et al. Health care-associated blood stream infections in adults: a reason to change the accepted definition of community-acquired infections. Ann Intern Med 2002 Nov; 137(10):791-797.
3. Hugonnet S, Sax H, Eggimann P, Chevrolet JC, Pittet D. Nosocomial bloodstream infection and clinical sepsis. Emerg Infect Dis 2004 Jan;10(1):76-81.

4. Nair PK, Vaz MS. Prevalence of carbapenem resistant Enterobacteriaceae from a tertiary care hospital in Mumbai, India. Microbiol Infect Dis 2013 Dec;3(4):207-210.

5. Karlowsky JA, Jones ME, Draghi DC, Thornsberry C, Sahm DF, Volturo GA. Prevalence and antimicrobial susceptibilities of bacteria isolated from blood cultures of hospitalized patients in the United States in 2002. Ann Clin Microbiol Antimicrob 2004 May;3:7.

6. Mehta M, Dutta P, Gupta V. Antimicrobial susceptibility pattern of blood isolates from a teaching hospital in north India. Jpn J Infect Dis 2005 Jun;58(3):174-176.

7. Jadhav S, Gandham N, Paul R, Misra RN, Ujagare MT, Angadi K, Vyawahare C. Bacteriological profile of septicaemia and antimicrobial susceptibility of isolates from tertiary care hospital in India. Res J Pharm Biol Chem Sci 2012 Oct-Dec;3(4):1100-1108.

8. Karunakaran R, Raja NS, Ng KP, Navaratnam P. Etiology of blood culture isolates among patients in a multidisciplinary teaching hospital in Kuala Lumpur. J Microbiol Immunol Infect 2007 Oct; 40(5):432-437.

9. Collee, JG.; Fraser, AG.; Marmion, BP.; Simmons, A. Tests for identification of bacteria. In: Mackie and McCartney practical medical microbiology. 14th ed. London: Churchill Livingstone; 1996. pp. 131-149.

10. Clinical and Laboratory Standards Institute (CLSI). Performance standards for antimicrobial susceptibility testing; Twenty-Sixth informational supplement. CLSI document M100-S26. Wayne (PA): CLSI; 2016. pp. 50-56.

11. Magiorakos AP, Srinivasan A, Carey RB, Carmeli $Y$, Falagas ME, Giske CG, Harbarth S, Hindler JF, Kahlmeter G, Olsson-Liljequist B, et al. Multidrug resistant, extensively drug-resistant and pan drug-resistant bacteria: an international expert proposal for interim standard definitions for acquired resistance. Clin Microbiol Infect 2012 Mar; 18(3):268-281.

12. Ali J, Kebede Y. Frequency of isolation and antimicrobial susceptibility pattern of bacterial isolates from blood culture, Gondar University teaching hospital, Northwest Ethiopia. Ethiop Med J 2008 Apr;46(2):155-161.

13. Gill MK, Sharma S. Bacteriological profile and antibiotic resistance pattern in blood stream infection in critical care units of a tertiary care hospital in North India. Indian J Microbiol Res 2016;3(3):270-274.

14. Arora U, Devi P. Bacterial profile of blood stream infections and antibiotic resistance pattern of isolates. JK Sci 2007 Jan;9(4):186-190.

15. Sharma M, Goel N, Chaudhary U, Aggarwal R, Arora DR. Bacteraemia in children. Indian J Pediatr 2002 Dec;69(12): 1029-1032.

16. Wasihun AG, Wlekidan LN, Gebremariam SA, Dejene TA, Welderufael AL, Haile TD, Muthupandian S. Bacteriological profile and antimicrobial susceptibility patterns of blood culture isolates among febrile patients in Mekelle Hospital, Northern Ethiopia. Springerplus 2015 Jul;4:314.

17. Dagnew M, Yismaw G, Gizachew M, Gadisa A, Abebe T, Tadesse T, Alemu A, Mathewos B. Bacterial profile and antimicrobial susceptibility pattern in septicemia suspected patients attending Gondar University Hospital, Northwest Ethiopia. BMC Res Notes 2013 Jul;6:283. 
18. Alam MS, Pillai PK, Kapur P, Pillai KK. Resistant patterns of bacteria isolated from bloodstream infections at a university hospital in Delhi. J Pharm Bioallied Sci 2011 Oct;3(4):525-530.

19. Anbumani N, Kalyani J, Mallika M. Original research distribution and antimicrobial susceptibility of bacteria isolated from blood cultures of hospitalized patients in a tertiary care hospital. Indian J Pract Dr 2008;5(2):1-7.

20. Gohel K, Jojera A, Soni S, Gang S, Sabnis R, Desai M. Bacteriological profile and drug resistance patterns of blood culture isolates in a tertiary care nephrourology teaching institute. Biomed Res Int 2014 Apr;2014:153747.
21. Gupta S, Kashyap B. Bacteriological profile and antibiogram of blood culture isolates from a tertiary care hospital of North India. Trop J Med Res 2016 Jul;19(2):94-99.

22. Singh AK, Venkatesh V, Singh RP, Singh M. Bacterial and antimicrobial resistance profile of bloodstream infections: a hospital based study. CHRISMED J Health Res 2014 Aug;1(3):140-144.

23. Nwadioha SI, Nwokedi EO, Kashibu E, Odimayo MS, Okwori EE. A review of bacterial isolates in blood cultures of children with suspected septicemia in a Nigerian. Afr J Microbiol Res 2010 Feb;4(4):222-225. 